\title{
Hyperbaric Oxygen Therapy in Managing Minimally Invasive Aesthetic Procedure Complications: A Report of Three Cases
}

\author{
Mendy Hatibie Oley $\mathbb{D}^{1-3}$ \\ Maximillian Christian Oley (iD ${ }^{3-5}$ \\ Ferra Olivia Mawu ${ }^{6}$ \\ Deanette Michelle R Aling (iD) ${ }^{3}$ \\ Muhammad Faruk (iD ${ }^{7}$ \\ 'Plastic Reconstructive and Aesthetic
} Surgery Division, Department of Surgery, Faculty of Medicine, Sam Ratulangi University, Manado, Indonesia; ${ }^{2}$ Plastic Reconstructive and Aesthetic Surgery Division, Department of Surgery, R. D. Kandou Hospital, Manado, Indonesia; ${ }^{3}$ Hyperbaric Centre, Siloam Hospital, Manado, Indonesia; ${ }^{4}$ Division of Neurosurgery, Department of Surgery, Faculty of Medicine, Sam Ratulangi University, Manado, Indonesia; ${ }^{5}$ Division of Neurosurgery, Department of Surgery, R. D. Kandou Hospital, Manado, Indonesia; ${ }^{6}$ Department of Dermatology and Venereology, Faculty of Medicine, Sam Ratulangi University, Manado, Indonesia; ${ }^{7}$ Department of Surgery, Faculty of Medicine, Hasanuddin University, Makassar, Indonesia
Introduction: Minimally invasive aesthetic procedures such as filler injections and thread lifts have gained popularity recently. Complications from these aesthetic procedures are difficult to avoid. This increasing public health concern requires a combination of effective therapeutic modalities. Hyperbaric oxygen therapy (HBOT) has generated favorable results in treating a diversity of wounds, inflammation, and infection.

Case Presentations: Three cases with complications arising from aesthetic procedures were described in this report. The patients were all female, with ages ranging from the latetwenties to mid-fifties. Two patients experienced complications from filler injections, one of which progressed to a parotid gland infection due to a placental extract filler while the other was caused by a hyaluronic acid filler. The third patient had notable excoriations and inflammation on both cheeks following a thread lift procedure. Alongside antibiotics and other symptomatic therapies, the patients received multiple, 90-minute HBOT sessions at 2.4 ATA over the course of one to two weeks. The wounds were frequently monitored to evaluate the healing progress.

Discussion: Complications from facial rejuvenation procedures can be disastrous. They expose the patient to the risk of developing vascular occlusions and skin infections that require prompt and effective treatment. Multiple treatment options (eg, frequently massaging the affected area, warm compresses, aspirin, and antibiotics) have been used to treat these complications. HBOT serves a valuable purpose in restoring adequate tissue perfusion in cases of filler-induced vascular occlusion and infection. Moreover, HBOT assists in restoring tissue injury and reducing inflammation following thread lift procedures.

Conclusion: HBOT has proven helpful as a treatment adjunct toward counteracting the effects of minimally invasive aesthetic procedures in several cosmetic-related cases.

Keywords: hyperbaric oxygen therapy, filler injection, thread lift, complications, aesthetic procedures

\section{Introduction}

The desire to stay young or mirror a certain image may bring more harm than good, especially when complications from aesthetic procedures are difficult to avoid. The use of minimally invasive techniques for aging-related conditions and facial corrections have been gaining popularity in recent years. In 2020, the American Society of Plastic Surgeons (ASPS) reported a 13.2 million total cosmetic minimallyinvasive cosmetic procedures, $174 \%$ increase in minimally-invasive cosmetic procedures since 2000 . Soft tissue fillers represent $25.68 \%$ of the 13.2 million of these
Correspondence: Mendy Hatibie Oley Plastic Reconstructive and Aesthetic Surgery Division, Department of Surgery, Faculty of Medicine, Sam Ratulangi University, Jalan Raya Tanawangko No. 56, Malalayang Satu Barat, Malalayang,

Manado, 95162, Indonesia

Fax +6243I-863786

Email mendy.hatibie@unsrat.ac.id 
procedures performed in the United States (US) annually. ${ }^{1}$ Minimally invasive procedures have also become popular recently in the Asian market, influenced by South Korean culture. Asian patients, like those in the United States, prefer non-surgical, minimally invasive treatment options and show concern about retaining a natural appearance. ${ }^{2}$ In terms of surgery and nonsurgical procedures, South Korea did nearly 6 times more than the global average. In 2011, 130.564 cosmetic procedures per 10.000 people were performed in South Korea, 78.665 of which were nonsurgical such as hyaluronic acid filler and botulinum toxin (popularly referred to as "botox") injections. ${ }^{3}$

Hyaluronic acid, placental extract, calcium hydroxylapatite, and poly-L-lactic acid are a few examples of commonly used fillers today. ${ }^{4}$ Dermal fillers are injected into the desired area to augment the skin and create a "revivedskin" look. ${ }^{5}$ However, administering these fillers poses the risk of complications, including infection and vascular occlusion, which, if left untreated, may progress to tissue necrosis. $^{6}$

Thread lifting involves a thread with barbs at the proximal end or threads without barbs configured in a meshlike form being embedded in the fascia. The most common thread used in this procedure is polydioxanone (PDO), considered one of the safest materials as it is absorbed within 6 months. $^{7}$ Complications such as bruising, swelling, facial asymmetry, and suture extrusion require immediate and effective treatment modalities that prevent further tissue damage and infection. ${ }^{7,8}$

The cellular inflammatory cascade and wound healing process is triggered by tissue hypoxia. Maintaining an oxygen pressure of at least $30 \mathrm{mmHg}$ in tissues is essential to provide an environment suitable for wound healing. Hyperbaric oxygen therapy (HBOT) is a safe and effective therapeutic option that facilitates the systematic repair of ischemic tissues by direct oxygen diffusion through the inhalation of $100 \%$ oxygen in a pressurized chamber. The benefits of HBOT have been reported in cases of post filler necrosis, vascular occlusion, crush injuries, difficult-toheal ulcers, hand-replantation, and deep skin infections. ${ }^{9}$ This report further describes the use of HBOT as adjunctive therapy in three cases of complications arising from aesthetic procedures.

\section{Case Presentation Case I}

A 55-year-old female patient presented with inflammation and redness of the right jaw (Figure 1A). The patient had received filler injections along her left and right jaw lines made from placental extract 2 weeks prior. Three days after the injection, she experienced pain and tenseness in her right jaw that prevented her from fully opening her mouth. No fever was

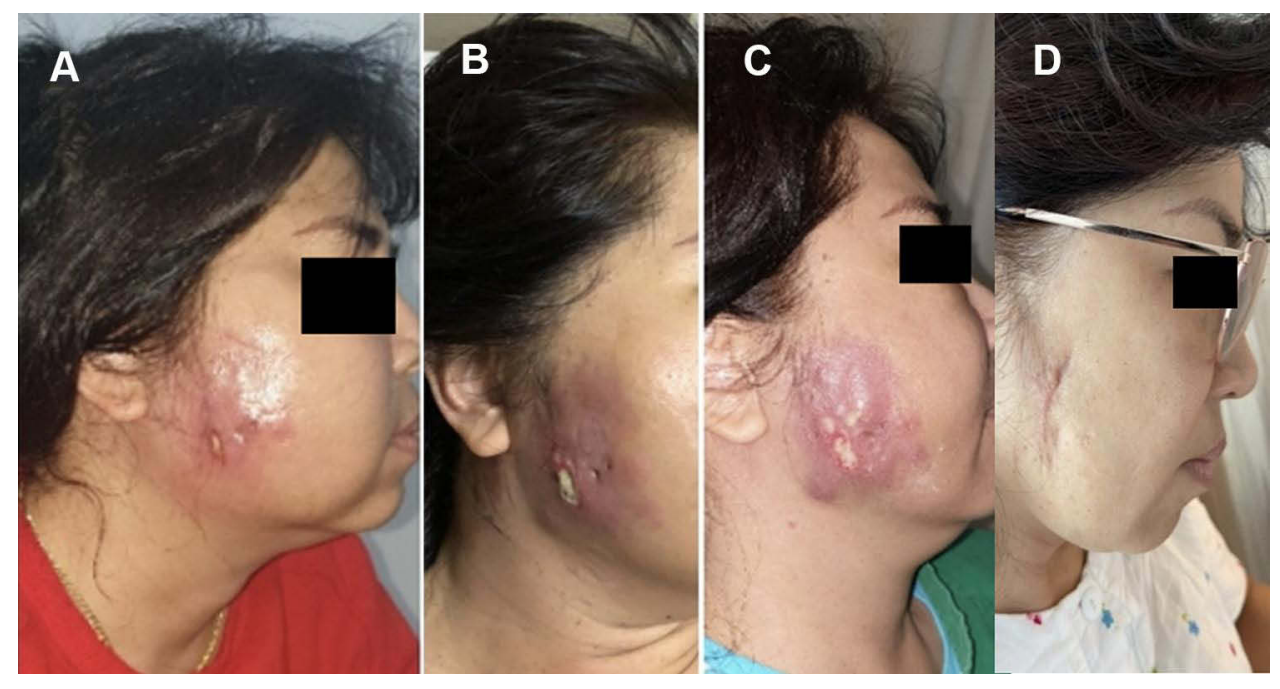

Figure I Sialadenitis caused by placenta-extract filler injections. (A) Inflamed right jaw prior to HBOT. (B) Slowly draining abscess and reduced swelling after three HBOT sessions. (C) After six HBOT sessions, the area of infection became more localized. (D) A completely healed wound at I-year post debridement. 
reported. Her social and medical histories were unremarkable. Physical examination revealed an inflamed right buccal region within the salivary gland area with a pus-filled core and an unclear margin. Laboratory results indicated a slightly elevated leukocyte count $(12,500$ per $\mathrm{uL}$ of blood), while the other parameters were within the normal limits. The patient was diagnosed with sialadenitis likely caused by a blockage of the salivary gland or duct from filler injections. Her treatments included daily debridement, IV broad-spectrum antibiotics for 7 days along with 7 consecutive, 90-minute HBOT sessions, once daily at 2.4 ATA (Figure 1B). The wound was clinically evaluated every 3 days. As the days progressed, the inflammation began to reside as the area of infection continued to shrink, leaving a once-blurred, now clear margin around the wound (Figure 1C). The wound fully healed within one year (Figure 1D).

\section{Case 2}

A 29-year-old female complained of red patches on her cheek and chin area. Two weeks prior to her visit, she received a thread lift procedure using barbed threads along her nasolabial folds and jawlines from an aesthetician at a local beauty clinic (Figure 2A). She experienced itchiness around the implanted area the day after the procedure and began scratching her face. A week later, she noticed an exposed thread on her right cheek and returned to the clinic but received no satisfactory explanation.
Consequently, she forcefully pulled the thread out herself. The patient had no remarkable medical or social history. Multiple small wounds with erythema were observed on the nasolabial folds and buccal regions. The laboratory results were unremarkable. We suspected that the patient exhibited a hypersensitivity reaction to the absorbable surgical suture used in the procedure, and this had progressed into a secondary skin infection caused by continuous scratching and the vigorous extraction of the thread from her right cheek. The patient received oral broadspectrum antibiotics for 7 days, nine 90-minute HBOT sessions at 2.4 ATA three times per week, and antihistamine tablets once daily. After one month, the wound exhibited less inflammation, improved vascularization, and presented a vibrant red color (Figure 2B).

\section{Case 3}

A 32-year-old female arrived at our clinic for a consultation regarding a nose implant. In her attempt for a higher nose tip, she had received injections of hyaluronic acid fillers into her nose a few weeks prior at a beauty clinic. Her medical and social histories were unremarkable. Inflammation with blanchable erythema on the nasal tip was evident upon inspection with slight tenderness upon palpation (Figure 3A). The laboratory results were unremarkable. The patient was diagnosed with post-filler vascular occlusion. The implant procedure was canceled due
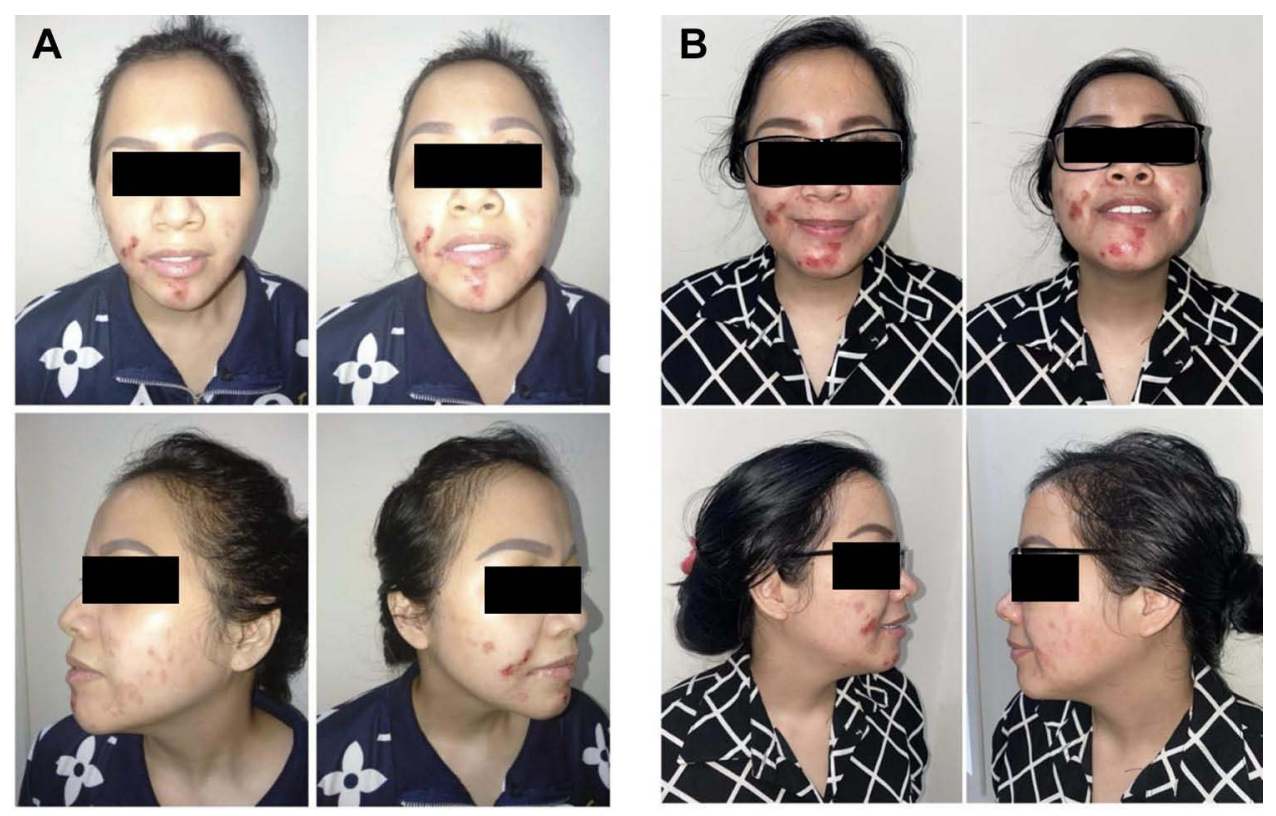

Figure 2 Post thread lift allergy leading to facial excoriations. (A) Multiple wounds on the nasolabial folds and jawlines before HBOT. (B) Inflammation resided and wounds turned bright red as vascularization improved after one month of HBOT. 


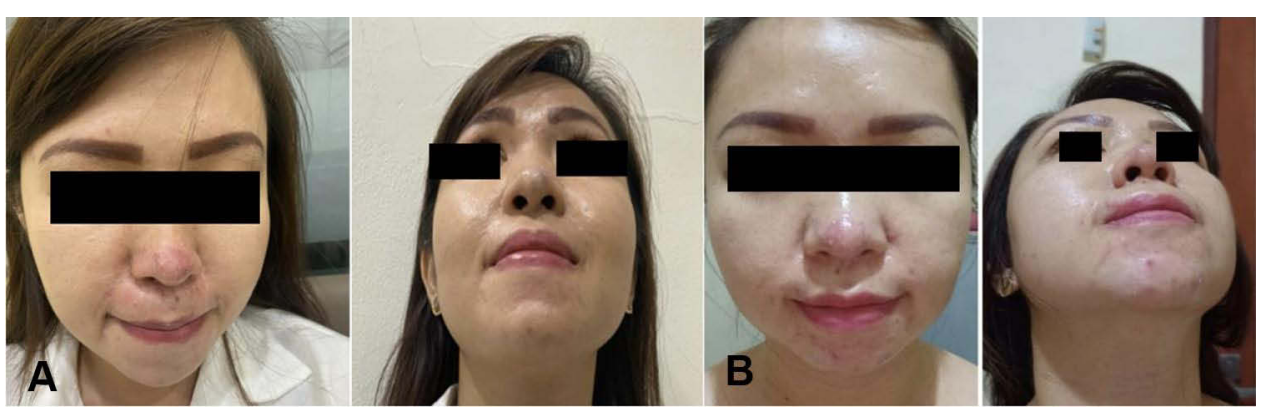

Figure 3 Post-filler vascular occlusion of the nasal tip. (A) Erythema and blanching of the nasal tip before HBOT. (B) Excoriations turned bright red as vascularization began to improve after one month of HBOT.

to the risk of infection from both filler and implant being present in the same area. 90 minute-HBOT sessions at 2.4 ATA were administered twice weekly to improve vascularization into the nasal region. One month later, the patient's nose appeared vibrant red with less discoloration indicating enhanced blood flow to the nasal area (Figure 3B).

\section{Discussion}

Complications from aesthetic facial procedures can be disastrous due to the risk of developing vascular occlusions and skin infections. As such, they require prompt and effective therapy solutions. By utilizing highly pressurized oxygen diffusion to hypoxic tissues to enhance the vascularization of the face and given its bactericidal effects, HBOT serves a beneficial purpose in the wound healing process.

The use of non-invasive facial rejuvenation techniques (eg, fillers) has gained popularity in recent years. Hyaluronic acid is favored among the other types of fillers as it is regarded as the safest, most effective filler for the facial region. Placental extracts are another form of facial filler. A study in Korea examined combinations of human fibroblasts and placental extract as facial augmentation fillers. The study found significant statistically fibroblast proliferation in the treatment group compared with the controls. ${ }^{10}$ Though being considered safe, the injection of facial fillers still poses risks for its recipients. ${ }^{11}$ In the Netherlands, the likely under-reported incidence of vascular obstruction from filler injection is 1:6600. ${ }^{12}$ Some vascular adverse effects are caused by the wrong injection site, excessive filler volume, improper indications, and incorrect injection techniques. ${ }^{5}$ The most common mechanism of vascular occlusion is an intravascular embolism, which is indicated by pain, blanching, livedo pattern, delayed capillary refill time, blue or greyish discoloration, and coolness of the occluded area. ${ }^{13}$ Glashofer et al divided the adverse effects of filler injections into two groups: early and delayed. The early effects included bruising, swelling, erythema at the injection site, hypersensitivity, and vascular compromise. The delayed effects included infection, as well as the formation of granulomas and biofilms. ${ }^{14}$

In recent years, HBOT has been gaining popularity in treating vascular compromise and deep skin infections due to its hyperoxygenation effect. ${ }^{15}$ Hyperoxygenation triggers local cell metabolism and promotes recovery of cellular function thereby increasing fibroblast proliferation, microvascular repair, and capillary angiogenesis. The release of reactive oxygen species (ROS) and vascular endothelial growth factor (VEGF) plays a significant role in this case. ${ }^{16}$ These help to improve microcirculation, promote wound healing, and control infections. ${ }^{17}$ Therefore, HBOT has been used as an adjunctive therapy option in several cases of vascular occlusion due to filler injections. ${ }^{11,14,18}$

In cases 1 and 3, HBOT was administered along with antibiotics to ease the infection of the parotid gland and the surrounding skin for patient 1 and to aid the vascularization of ischemic tissue on the nose of patient 3. Several studies on the body's reaction to filler injections have been published. One case of dermal filler on the buccal region had a delayed onset and diffused swelling that occurred years after the procedure, triggered by a periodontal surgery. ${ }^{19}$ Another delayed case of dermal fillers was the manifestation of a small parotid mass followed by an eyebrow mass two months after the parotidectomy. ${ }^{20}$ Therefore, it is quite common for the salivary gland to be affected during this procedure. HBOT has proven effective in treating multiple cases of filler rhinoplasty ${ }^{21}$ and tissue necrosis. ${ }^{22}$ Unfortunately, to date, there is no 
protocol for how many sessions needed to treat infections. Therefore, the number of HBOT sessions is determined upon clinical evaluation by the physician.

Another method of facial rejuvenation is thread lifting, which has garnered attention in recent years due to being minimally invasive with results lasting up to 5 years after the procedure. ${ }^{7}$ Polydioxanone (PDO) threads are commonly used in this procedure and are absorbed by the body over a 4 to 6-month period. Many complications arise from this technique including thread exposure, dimpling, parotid gland injury, inflammation, and facial asymmetry. Anh et al reported a case of multiple palpable masses on the cheeks after 3 courses of acupoint embedding therapy (AET) with PDO over 2 years. ${ }^{23}$ Wang presented two cases of thread lifting complications (thread extrusion, skin dimpling). ${ }^{24}$ Patient 2 received HBOT sessions to reduce the inflammation from an allergic reaction and restore the tissues injured by the implanted barbed thread.

The recommended treatments for vascular compromise include firmly massaging the affected area, warm compression, aspirin, hyaluronidase injection and, specifically post hyaluronic acid injection, antibiotics and HBOT as an added treatment option. ${ }^{13}$ HBOT is utilized as an adjunctive treatment to facilitate tissue repair. Given its antiinflammatory and anti-bacterial effects, HBOT is an effective means of treating complications arising from aesthetic procedures. Follow-up visits are necessary to ensure the effectiveness of the treatment provided. The number of visits depends on the clinical evaluation of the affected area during the patient's last visit.

\section{Conclusion}

The effects of HBOT in combination with antibiotics, antiinflammatory and antihistamine agents in managing complications from minimally invasive aesthetic procedures are favorable. HBOT is a supplemental therapy that effectively delivers oxygen systemically to maintain tissue viability during such complications.

\section{Ethics Approval and Informed Consent}

All procedures performed in this report involving human participants were in accordance with the ethical standards of the institutional and/or national research committee and with the 1964 Helsinki declaration and its later amendments or comparable ethical standards. Written informed consent has been provided by all the patients to have the case details and any accompanying images published. The study is exempt from ethical approval in our institution.

\section{Author Contributions}

All authors made a significant contribution to the work reported, whether that is in the conception, study design, execution, acquisition of data, analysis and interpretation, or in all these areas; took part in drafting, revising or critically reviewing the article; gave final approval of the version to be published; have agreed on the journal to which the article has been submitted; and agree to be accountable for all aspects of the work.

\section{Disclosure}

The authors have no financial conflicts of interest regarding the publication of this article.

\section{References}

1. American Society of Plastic Surgeons. Plastic surgery statistics report; 2020. 1-26. Available from: https://www.plasticsurgery.org/ documents/News/Statistics/2020/plastic-surgery-statistics-full-report2020.pdf. Accessed December 30, 2021.

2. Chiu A, Mariwalla K, Hui-Austin A, Narurkar V, de la Guardia C. Understanding the female Asian American facial aesthetic patient. $J$ Drugs Dermatol. 2019;18:633-641.

3. Vorisek RM. Cross-Cultural Comparison of Cosmetic Procedures in Latin America and East Asia. University of Mississippi; 2017.

4. van Loghem J, Funt D, Pavicic T, et al. Managing intravascular complications following treatment with calcium hydroxylapatite: an expert consensus. J Cosmet Dermatol. 2020;19:2845-2858. doi:10.1111/jocd. 13353

5. Haneke E. Managing complications of fillers: rare and not-so-rare. $J$ Cutan Aesthet Surg. 2015;8:198-210. doi:10.4103/0974-2077.172191

6. Tracy L, Ridgway J, Nelson JS, Lowe N, Wong B. Calcium hydroxylapatite associated soft tissue necrosis: a case report and treatment guideline. J Plast Reconstr Aesthet Surg. 2014;67:564-568. doi:10.1016/j.bjps.2013.08.008

7. Wu WTL, Mendelson B. Invited discussion on: mesh suspension thread for facial rejuvenation. Aesthetic Plast Surg. 2020;44:775779. doi:10.1007/s00266-020-01670-w

8. Lee H, Yoon K, Lee M. Outcome of facial rejuvenation with polydioxanone thread for Asians. J Cosmet Laser Ther. 2018;20:189-192. doi:10.1080/14764172.2017.1400167

9. Yamada N, Toyoda I, Ogura S. Hyperbaric oxygen therapy in the management of severe soft tissue injuries. In: Shinomiya N, Asai Y, editors. Hyperbaric Oxygenation Therapy. Singapore: Springer Singapore; 2020:81-104. doi:10.1007/978-981-13-7836-2

10. Oh EJ, Kim TK, Shin JH, Choi JH, Chung HY. Biologic filler using human fibroblasts and placenta extracts. $J$ Craniofac Surg. 2011;22:1557-1560. doi:10.1097/SCS.0b013e31822e5c5e

11. Bravo BS, Balassiano LK, Da Rocha CR, et al. Delayed-type necrosis after soft-tissue augmentation with hyaluronic acid. $J$ Clin Aesthet Dermatol. 2015;8:42-47.

12. Schelke L, Decates T, Kadouch J, Velthuis P. Incidence of vascular obstruction after filler injections. Aesthetic Surg J. 2020;40:NP457NP460. doi:10.1093/asj/sjaa086 
13. King M, Walker L, Convery C, Davies E. Management of a vascular occlusion associated with cosmetic injections. $J$ Clin Aesthet Dermatol. 2020;13:E53-E58.

14. Beleznay K, Humphrey S, Carruthers JDA, Carruthers A. Vascular compromise from soft tissue augmentation: experience with 12 cases and recommendations for optimal outcomes. $J$ Clin Aesthet Dermatol. 2014;7:37-43.

15. Hong WT, Kim JI, Kim SW. Minimizing tissue damage due to filler injection with systemic hyperbaric oxygen therapy. Arch Craniofacial Surg. 2019;20:246-250. doi:10.7181/acfs.2019.00059

16. Oley MH, Oley MC, Durry MF, Adam RN, Gunawan DF, Faruk M. Hyperbaric oxygenation therapy. Am Rev Respir Dis. 1991;144:1414-1421. doi:10.1164/ajrccm/144.6.1414

17. Oley MH, Oley MC, Noersasongko AD, et al. Effects of hyperbaric oxygen therapy on vascular endothelial growth factor protein and mRNA in crush injury patients: a randomized controlled trial study. Int J Surg Open. 2021;29:33-39. doi:10.1016/j.ijso.2021.01.003

18. DeLorenzi C. Complications of injectable fillers, part 2: vascular complications. Aesthetic Surg J. 2014;34:584-600. doi:10.1177/ $1090820 \times 14525035$
19. Florin W, Mandel L. Foreign body reaction to facial dermal fillers: case report. J Oral Maxillofac Surg. 2012;70:2352-2355. doi:10.1016/j.joms.2011.11.008

20. Coughlin A, Gray ML, Westra WH, Teng MS, Rosenberg JD. Dermal filler presenting as parotid mass: a case report. Head Neck Pathol. 2021;15:638-641. doi:10.1007/s12105-020-01197-1

21. Tsai, Mei-Chih, Te-Chun Hsia, Yen-Wei Han, Huei-Ru Yau Han-Dau $\mathrm{Wu}$ and Yu-ya Lin. "Successful Hyperbaric Oxygen Therapy In Complications Of Fillers Rhinoplasty-Cases Report." The Internet Journal of Alternative Medicine 9 (2014). Available from: https:// ispub.com/IJAM/9/1/14758.

22. Kruize RGF, Teguh DN, van Hulst RA. Hyperbaric oxygen therapy in hyaluronic acid filler-induced dermal ischemia. Dermatolog Surg. 2020;46:1755-1757. doi:10.1097/DSS.0000000000002109

23. Ahn SK, Choi HJ. Complication after PDO threads lift. J Craniofac Surg. 2019;30:e467-e469. doi:10.1097/SCS.0000000000005644

24. Wang C-K. Complications of thread lift about skin dimpling and thread extrusion. Dermatol Ther. 2020;33:e13446-e13446. doi:10.1111/dth. 13446

\section{Publish your work in this journal}

Clinical, Cosmetic and Investigational Dermatology is an international, peer-reviewed, open access, online journal that focuses on the latest clinical and experimental research in all aspects of skin disease and cosmetic interventions. This journal is indexed on CAS.
The manuscript management system is completely online and includes a very quick and fair peer-review system, which is all easy to use. Visit http://www.dovepress.com/testimonials.php to read real quotes from published authors. 